\title{
Retrospective Cross-Sectional Analysis of Treatment Processes of Children Diagnosed with Obsessive Compulsive Disorder
}

\author{
Halil Kara $^{1}$ (D) S. Burak Açıkel ${ }^{2}$ \\ 1 Aksaray University Medical Faculty, Department of Child and Adolescent Psychiatry, Aksaray, Turkey. \\ 2 Dr. Sami Ulus Maternity and Children's Health and Diseases Training and Research Hospital, Department of Child and Adolescent Psychiatry, Ankara, Turkey.
}

\begin{abstract}
Background: Obsessive-compulsive disorder (OCD) is a severe, common and most often chronically debilitating disorder characterized by repetitive, ritualistic, and distressing thoughts, ideas, and behaviors. In this retrospective study, we have presented patients who have admitted to our clinic and was diagnosed with obsessive-compulsive disorder and their baseline and after-treatment symptom severity.
\end{abstract}

Methods: Participants who admitted to Aksaray University Faculty of Medicine Department of Child Adolescent Psychiatry were evaluated. Medical records were obtained by previous clinical evaluation and scaled scores for this retrospective study. The Schedule for Affective Disorders and Schizophrenia for School Age Children- Present and Lifetime Version, DSM-5 (K-SADS-PL) was applied to every patient at first session of treatment. Symptom severity of OCD was recorded according to Children's YaleBrown Obsessive-Compulsive Scale.

Results: 73 patients were included in the study. $49.3 \%$ was male, $50.7 \%$ was female. $52.1 \%$ of patients have one or more comorbid disorders. All patients were using at least one pharmacological agent. There was a significant difference between baseline and eight weeks after treatment CY-BOCS total scores $(\mathrm{p}<0.001)$.

Conclusions: As a conclusion, pediatric OCD is a common and serious psychiatric disorder and SSRI medication is an effective choice. Compliance to treatment and regular follow-up is important for improvement.

Key words: Pediatric, Obsessive-Compulsive Disorder, Adolescent, Treatment.

\section{INTRODUCTION}

Obsessive-compulsive disorder (OCD) is a severe, common and most often chronically debilitating disorder characterized by repetitive, ritualistic, and distressing thoughts, ideas, and behaviors (1). Research suggests approximately $50 \%$ of all cases have their onset in childhood or adolescence (2). Obsessions and compulsions in children are more likely to change/evolve as well as wax and wane, compared to the course of the disorder in adults. An epidemiological study conducted in USA reported that $21 \%$ of OCD cases had onset by age 10 (3). Pediatric OCD appears to be more common in males than in females, in contrast to adults where the male-female ratio of OCD is approximately 1:1 (4). 
Treatment of OCD in children and adolescents include cognitive behavioral therapy (CBT) and selective serotonergic reuptake inhibitors (SSRIs) (5). For mild to moderate cases of pediatric OCD, CBT is suggested as first-line treatment. An SSRI can also be used as first-line treatment in cases of patient and family preference or if CBT is not suitable. For more severe cases, a combination of an SSRI and CBT is suggested as first-line treatment. The SSRI may be needed to decrease the OCD and associated anxiety symptoms to a level where CBT can be effective (6). Medications with demonstrated efficacy as monotherapy in children and adolescents with OCD include three selective serotonin reuptake inhibitors (SSRIs; fluoxetine, fluvoxamine, and sertraline) and a serotonergic, tricyclic antidepressant, clomipramine. One of the SSRIs as firstline choice of medications is suggested.

In this retrospective study, we have presented patients who admitted to our clinic and were diagnosed with obsessive-compulsive disorder and the patients' baseline and after-treatment symptom severity.

\section{MATERIALS AND METHODS}

Study protocol was approved by the Non-Interventional Ethics Committee of Aksaray University (Date: 30.04.2020, No: 2020/05-01).

Participants who admitted to Aksaray University Faculty of Medicine Department of Child Adolescent Psychiatry were evaluated retrospectively. Study protocol was approved by the Non-Interventional Ethics Committee of Aksaray University. Medical records were obtained by previous clinical evaluation and scaled scores for this retrospective study.

Patients who admitted to the outpatient child and adolescent psychiatry clinic, were diagnosed as obsessive compulsive disorder (OCD) and followed in the same clinic were included. Sociodemographic data were obtained from hospital records. The socioeconomic status was classified into three groups as low, moderate and high and scored according to parental education level, parental employment status, monthly household income level, the presence of a washing machine, dishwasher, computer and other technological devices in their home, and whether or not the child benefitted from education. Psychosocial stressors were accepted as difficult life conditions such as separated parents, mother or father working far from home, exposure to peer bullying, exposure to physical violence and loss of a loved one.
The Schedule for Affective Disorders and Schizophrenia for School Age Children-Present and Lifetime Version, DSM-5 (K-SADS-PL) was applied to every patient at first session of treatment. The Kiddie-schedule for affective disorders and schizophrenia for school-age childrenpresent and lifetime version (K-SADS-PL-DSM5), which is a semi-structured interview, was developed by Kauffman et al. (13) The initial reliability and validity of the data obtained with this scale in Turkey was carried out by Ünal et al. (14)

When the first Covid-19 case was recorded in Turkey, precautions against the pandemic were rapidly implemented. One of these precautions was restriction of non-emergency patients presenting at hospitals. Therefore, as recommended, the patients being followed up in outpatient clinics were contacted by telephone, and patient evaluations and follow-up after the outbreak of the pandemic were carried out as described (9). Within the context of the Covid-19 pandemic precautions, procurement of medications was facilitated for patients so that they could obtain their regular drug treatments from pharmacies without seeing a doctor.

Symptom severity of OCD was recorded according to Children's Yale-Brown Obsessive-Compulsive Scale (CY-BOCS). In the follow-up, when a diagnosis of OCD was considered, CY-BOCS was applied to patients and follow-up was done accordingly. CY-BOCS, developed by Goodman et al. in 1986, is a semi-structured questionnaire based on clinical interviews (15). The patients were evaluated according to the clinical judgement of the interviewer based on the information given by the child and the parents. A total severity score was obtained from the obsession and compulsion severity points. An interobserver reliability study of the scale in a Turkish sample was published by Yücelen et al (16).

Statistical analysis was performed using the Statistical Package for Social Sciences (SPSS) version 23.0 for Windows software (SPSS Inc., Chicago, IL, USA). A value of $p<0.05$ was accepted as statistically significant.

\section{RESULTS}

773 patients were included the study. $49.3 \%$ was male, $50.7 \%$ was female. The mean age of the population was 12.82 ( $\mathrm{MIN}=7, \mathrm{MAX}=17$ ). $19.2 \%$ of the patients had low, $58.9 \%$ had moderate and $21.9 \%$ had high socioeconomic status. The ratio of having a psychosocial stress factor before starting treatment was $26 \%$. The mean duration between the beginning of obsessive-compulsive 
symptoms and the time of diagnosis was 11.27 months ( $\mathrm{MIN}=4$ months, $\mathrm{MAX}=36$ months). The mean followup duration after diagnosis was 6.39 months $(\mathrm{MIN}=3$ months, MAX=16months)

$52.1 \%$ of patients have one or more comorbid disorders. The comorbidity distribution data were as follows: $11 \%$ generalized anxiety disorder, $9.6 \%$ attention deficit hyperactivity disorder, $6.8 \%$ tic disorder, $6.8 \%$ social anxiety disorder, $2.7 \%$ panic disorder, $\% 2.7$ major depressive disorder, $2.7 \%$ specific learning disorder, $2.7 \%$ separation anxiety disorder, $2.7 \%$ trichotillomania, $2.7 \%$ oppositional defiant disorder, $1.4 \%$ nocturnal enuresis.

All the patients were given at least one pharmacological agent. In the monotherapy group, $66 \%$ of patients used fluoxetine $10-60 \mathrm{mg} /$ day, $28 \%$ used sertraline 50-150 $\mathrm{mg} /$ day and $6 \%$ used clomipramine $25-75 \mathrm{mg} /$ day. The combined pharmacological treatment ratio was $31.5 \%$. In the combined treatment group, $21.9 \%$ of patients used antipsychotics (15.1\% risperidone, 6.8\% aripiprazole), $5.5 \%$ methylphenidate, $4.1 \%$ atomoxetine. $61.7 \%$ fluoxetine, $30.1 \%$ sertraline, and $8.2 \%$ clomipramine.

There was a significant difference between baseline $(\mathrm{Mean}=27.10)$ and eight weeks after treatment $($ Mean=14.16) CY-BOCS total scores $(p<0.001)$. When severity scores were compared, there was a significant difference between baseline $(\mathrm{Mean}=3.7)$ and 8-week $($ Mean=1.9) severity scores $(\mathrm{p}<0.001)$. There was a significant difference between baseline CY-BOCS obsession scores $(\mathrm{p}=0.026)$ and CY-BOCS compulsion scores $(p=0.035)$ between the monotherapy group and the combined therapy group. At the end of the 8-week treatment period, there was no significant difference between groups' CY-BOCS total scores $(\mathrm{p}=0.809)$.

\section{DISCUSSION}

In this study, we have presented retrospective data about patients diagnosed as OCD in our clinic. We have mentioned patients' sociodemographic characteristics, distribution of medical therapy choice and baseline and 8-week symptom severity.

Selective serotonin reuptake inhibitors (SSRIs) are the first-line medication of choice because of their efficacy and generally well tolerated side effect profile. SSRIs shown to be effective and safe in this population include fluoxetine, fluvoxamine, and sertraline (6). In a meta-analysis serotonergic antidepressants (SSRIs and clomipramine) are reported to reduce OCD symptoms compared to placebo. An overall effect size for medication versus placebo was 0.46 (standardized mean difference; 95\% CI 0.37-0.55), equivalent to approximately four points on CY-BOCS (7). Similarly, we have found significant improvement in our patients. It was reported in the literature that more than half of the pediatric patients with OCD have been found to have at least one comorbid psychiatric disorder (8). Similar to this ratio, $52.1 \%$ of the patients in the present study have one or more comorbid disorders.

Specific SSRIs tested in clinical trials of pediatric OCD, all with positive results, include sertraline(5), fluoxetine(9) and fluvoxamine(10). In a clinical trial, 187 children and adolescents aged 13 to 17 years were randomly assigned to use sertraline (up to $200 \mathrm{mg} /$ day) or placebo (11). Patients treated with sertraline showed greater improvement compared placebo-treated patients in rate of response ( $42 \%$ vs $26 \%$ ) and reduction of OCD symptoms. Similar to these results, the most common medical agents used in the present study were fluoxetine and sertraline, respectively. Significant improvement was achieved with these agents.

Our study has certain limitations. The data was collected in one center and this is clearly a limitation when the results are supposed to represent a whole population. The follow-up period was limited to 8-weeks, thus, no long-term follow-up was carried out. Also, Comparison the effects of monotherapy and combined therapy were not compared because of the design of study.

As a conclusion, pediatric OCD is a common and serious psychiatric disorder and SSRI medication is an effective choice. Compliance to treatment and regular follow-up is important for improvement.

\section{Declarations}

The authors received no financial support for the research and/or authorship of this article. There is no conflict of interest

Study protocol was approved by the Non-Interventional Ethics Committee of Aksaray University (Date: 30.04.2020, No: 2020/05-01). 


\section{REFERENCES}

1. APA. The Diagnostic and Statistical Manual of Mental Disorders: DSM 5 [Internet]. 1. American Psychiatric Association; 2013. Available from: http://books.google.de/books?hl=en\&lr=\&id=_ VzzAgAAQB A \& \& o $i=f n d \& p g=P T 2 \& d q=d i a g n o s t i c+$ s y s t e m + of + m e n t a $1+d$ i s o r d e r s \& o t s = oTWum7IZ11\&sig=2WJpMGqUijr9ShY7uaifRZwKslM

2. Janowitz D, Grabe HJ, Ruhrmann S, Ettelt S, Buhtz F, Hochrein A, et al. Early onset of obsessive-compulsive disorder and associated comorbidity. Depress Anxiety [Internet]. 2009;26(11):1012-7. Available from: http: / / www.ncbi.nlm.nih.gov/pubmed/19691024

3. Kessler RC, Berglund P, Demler O, Jin R, Merikangas KR, Walters EE. Lifetime prevalence and age-of-onset distributions of DSM-IV disorders in the National Comorbidity Survey Replication. Arch Gen Psychiatry [Internet]. 2005 Jun;62(6):593-602. Available from: http:/ / www.ncbi.nlm.nih.gov/pubmed / 15939837

4. Eichstedt JA, Arnold SL. Childhood-onset obsessive-compulsive disorder: a tic-related subtype of OCD? Clin Psychol Rev [Internet]. 2001 Feb;21(1):137-57. Available from: http://www.ncbi.nlm.nih. gov/pubmed/11148894

5. Pediatric OCD Treatment Study (POTS) Team. Cognitive-behavior therapy, sertraline, and their combination for children and adolescents with obsessive-compulsive disorder: the Pediatric OCD Treatment Study (POTS) randomized controlled trial. JAMA [Internet]. 2004 Oct 27;292(16):1969-76. Available from: http://www.ncbi.nlm.nih.gov/ pubmed/15507582

6. Practice parameter for the assessment and treatment of children and adolescents with obsessive-compulsive disorder. J Am Acad Child Adolesc Psychiatry [Internet]. 2012 Jan;51(1):98-113. Available from: http: / / www.ncbi.nlm.nih.gov/pubmed/22176943

7. Geller DA, Biederman J, Stewart SE, Mullin B, Martin A, Spencer $\mathrm{T}$, et al. Which SSRI? A meta-analysis of pharmacotherapy trials in pediatric obsessive-compulsive disorder. Am J Psychiatry [Internet]. 2003 Nov;160(11):1919-28. Available from: http:/ / www.ncbi.nlm.nih. gov/pubmed/14594734

8. Douglass HM, Moffitt TE, Dar R, McGee R, Silva P. Obsessivecompulsive disorder in a birth cohort of 18-year-olds: prevalence and predictors. J Am Acad Child Adolesc Psychiatry [Internet]. 1995 Nov;34(11):1424-31. Available from: http://www.ncbi.nlm.nih.gov/ pubmed/8543509

9. Liebowitz MR, Turner SM, Piacentini J, Beidel DC, Clarvit SR, Davies SO, et al. Fluoxetine in children and adolescents with OCD: a placebocontrolled trial. J Am Acad Child Adolesc Psychiatry [Internet]. 2002 Dec;41(12):1431-8. Available from: http://www.ncbi.nlm.nih.gov/ pubmed / 12447029

10. Riddle MA, Reeve EA, Yaryura-Tobias JA, Yang HM, Claghorn JL, Gaffney G, et al. Fluvoxamine for children and adolescents with obsessive-compulsive disorder: a randomized, controlled, multicenter trial. J Am Acad Child Adolesc Psychiatry [Internet]. 2001 Feb;40(2):222-9. Available from: http:/ / www.ncbi.nlm.nih.gov/ pubmed/11211371

11. March JS, Biederman J, Wolkow R, Safferman A, Mardekian J, Cook $\mathrm{EH}$, et al. Sertraline in children and adolescents with obsessivecompulsive disorder: a multicenter randomized controlled trial. JAMA [Internet]. 1998 Nov 25;280(20):1752-6. Available from: http:/ / www.ncbi.nlm.nih.gov/pubmed / 9842950 